\title{
Reliability of coronal plane rotation measurements in the medial column of the foot: a cadaveric study
}

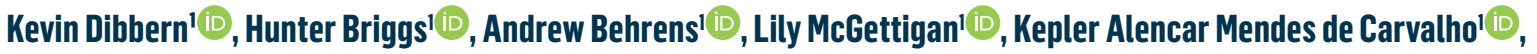 \\ Nacime Salomao Barbachan Mansur ${ }^{1,3}$, Matthieu Lalevée ${ }^{1,2}$ (D) , César de César Netto ${ }^{1} \mathbb{D}$ \\ 1. University of Iowa, Carver College of Medicine, Department of Orthopedics and Rehabilitation, Iowa City, IA, USA. \\ 2. Department of Orthopedic Surgery, Rouen University Hospital, Rouen, France. \\ 3. Federal University of São Paulo, Paulista School of Medicine, Department of Orthopedics and Traumatology, São Paulo, SP, Brazil.
}

\begin{abstract}
Objective: To assess interobserver reliability of previously described coronal plane rotation measurements of medial column bones and to assess their ability to accurately quantify changes in rotational profile.

Methods: Two cadaveric below-knee specimens were implanted with pins in each bone of the medial column. Weight-bearing computed tomography (CT) scans were acquired in a simulated standing position under neutral, supinated, and pronated conditions. For each specimen and condition, 2 observers measured the coronal plane rotation of the navicular, medial cuneiform, first metatarsal base, shaft, and head, and proximal phalanx of the hallux as previously described. The rotation of each pin was measured relative to the ground in the coronal plane for each condition. These measurements were defined as benchmarks for the rotational profile of each bone. The correlation between these benchmarks and direct bone measurements was then assessed. Intraclass correlation coefficient was used to assess interobserver reliability. Pearson's coefficient was used to evaluate correlations.
\end{abstract}

Results: The interobserver reliability of direct bone measurements ranged from 0.98 to 0.99 . Correlations between pin rotation and direct measurements ranged from $\rho=0.87$ to 0.99 across the neutral, supinated, and pronated conditions.

Conclusion: Coronal plane rotation measurements of medial column bones described in this study are reliable tools.

Level of Evidence III; Case-Control Study.

Keywords: Cadaver; Hallux valgus; Metatarsal bones; Pronation; Weight-bearing.

\section{Introduction}

The coronal plane rotational profile of medial column bones in the foot is a recent focus of attention ${ }^{(1-6)}$. Previously, understanding of foot pathologies was limited by conventional 2-dimensional radiographs and bone superimpositions ${ }^{(7-9)}$. Therefore, alignment of the tarsal bones in the coronal plane has been largely disregarded. Recently, several weight-bearing computed tomography (WBCT) studies have identified the presence of hyperpronation of the first ray relative to the ground in hallux valgus ${ }^{(1,2,6,10-12)}$. Subsequently, Conti et al. ${ }^{(13)}$ showed that surgical correction of this hyperpronation in hallux valgus decreased recurrence and improved patient-reported outcomes. Beyond hallux valgus deformity, assessing the tarsal bones in the coronal plane in other foot conditions, such as progressive collapsing foot deformity, cavovarus, and clubfoot, has shown promise for improving understanding and treatment ${ }^{(14-17)}$.

Most of the measurements described to assess the rotational profile of the medial column in the coronal plane only assess rotation at the level of the first metatarsal head ${ }^{(1,2)}$. Saltzman et al. ${ }^{(18)}$ on conventional radiographs and later Kim et al. ${ }^{(6)}$ on WBCT scans described measurements to assess coronal plane rotation of the first metatarsal head relative to the ground. However, these measurements assess not only the rotational profile of the first metatarsal but also the aggregate rotation of each bone in the medial column ${ }^{(5)}$. There-
Study performed at the University of lowa, Carver College of Medicine, Department of Orthopedics and Rehabilitation, lowa City, IA, USA.

Correspondence: César de César Netto. Department of Orthopaedic and Rehabilitation, University of lowa, Carver College of Medicine. 200 Hawkins Dr, John PappaJohn Pavillion (JPP). Room 01066, Lower Level. lowa City, IA, 52242 - United States. E-mail: cesar-netto@uiowa.edu. Conflicts of Interest: none. Source of funding: none. Date received: November 19, 2021. Date accepted: December 09, 2021. Online: December 20, 2021.
How to cite this article: Dibbern $\mathrm{K}$, Briggs $\mathrm{H}$, Behrens A, McGettigan L, Carvalho KAM, Mansur NSB, et al. Reliability of coronal plane rotation measurements in the medial column of the foot: a cadaveric study. J Foot Ankle. 2021;15(3):252-8. 
fore, hyperpronation present in hallux valgus deformity could originate from anywhere in the entire medial column, from the navicular to the first metatarsal. Understanding the origin of this hyperpronation could both improve comprehension of pathogenesis and guide surgical treatment.

Schmidt et al. ${ }^{(19)}$ were the first to describe coronal plane rotation measurements for each bone in the medial column. However, neither the interobserver reliability of these measurements nor their ability to quantify real changes in bone rotation in the coronal plane (pronation or supination) has been evaluated.

The primary objective of our study was to assess the interobserver reliability of these measurements along the medial column. The secondary objective was to assess the accuracy of these measurements in quantifying coronal plane rotation.

\section{Methods}

Institutional Review Board approval was obtained (IRB\# 201904825).

Two fresh frozen below-knee specimens without deformity or disease were obtained. The proximal 5 centimeters of the leg were prepared by removing the surrounding soft tissue to enable potting of the tibial plateau with bone cement. This apparatus provided the support to hold the specimens in a vertical plantigrade position in a radiolucent loading frame. These same specimens had been used in a previous study of instability of the distal tibiofibular syndesmosis, but the hindfoot, midfoot, and forefoot remained intact. The potted specimens were placed in the frame where a vertical load of 80 lbs. (356 N) was applied to simulate a double-legged stance with the tibia perpendicular to the floor (Figure 1). The frame holder was placed in a WBCT scanner (Hi-Rise, CurveBeam $^{\circledR}$ ) and foot acquisitions were obtained using a metal artifact reduction algorithm. Specimens were thawed for more than 24 hours before the acquisitions.

We implanted a 2-mm diameter pin in each bone of the medial column (one in the navicular, one in the medial cuneiform, one in the first metatarsal, and one in the proximal phalanx of the hallux) and ensured they were not torqued to remain straight for each specimen. After pin placement, each bone underwent 3 WBCT scans, one in each of the neutral, supinated, and pronated conditions (Figure 2).

The following measurements were performed using a dedicated software (CubeVue ${ }^{\mathrm{TM}}$, CurveBeam, LLC, Warrington, PA, USA) and after having aligned our sagittal plane on the longitudinal axis of the first metatarsal in the axial view. Navicular, medial cuneiform, first metatarsal base, and proximal phalanx of the hallux rotations were measured in accordance with the techniques described by Schmidt et al.(19). First metatarsal head rotation was additionally measured in accordance with the definitions proposed by Steadman et al. ${ }^{(4)}$, Kim et al. ${ }^{(6)}$, and Saltzman et al. ${ }^{(18)}$. We additionally proposed a new definition to assess first metatarsal rotation in the coronal plane at the midshaft level.

\section{Navicular rotation}

The navicular rotation was measured as the angle between the floor and the widest mediolateral distance of the bone, at a level just distal to the talonavicular joint ${ }^{(19)}$ (Figure 3A).

\section{Medial cuneiform rotation}

The medial cuneiform rotation was measured just proximal to the most proximal aspect of the first tarsometatarsal joint and was defined as the angulation between the vertical line and a bisecting line of an angle formed by tangent lines to the medial and lateral surfaces of the medial cuneiform ${ }^{(19)}$ (Figure 3B).

\section{First metatarsal base rotation}

The first metatarsal base coronal rotation was measured at a level just distal to the most distal aspect of the first tarsometatarsal joint. The rotation was defined as the angle between the floor and a bisecting line of the angle formed by tangent lines to the medial and lateral surfaces of the proximal first metatarsal(19) (Figure 3C).

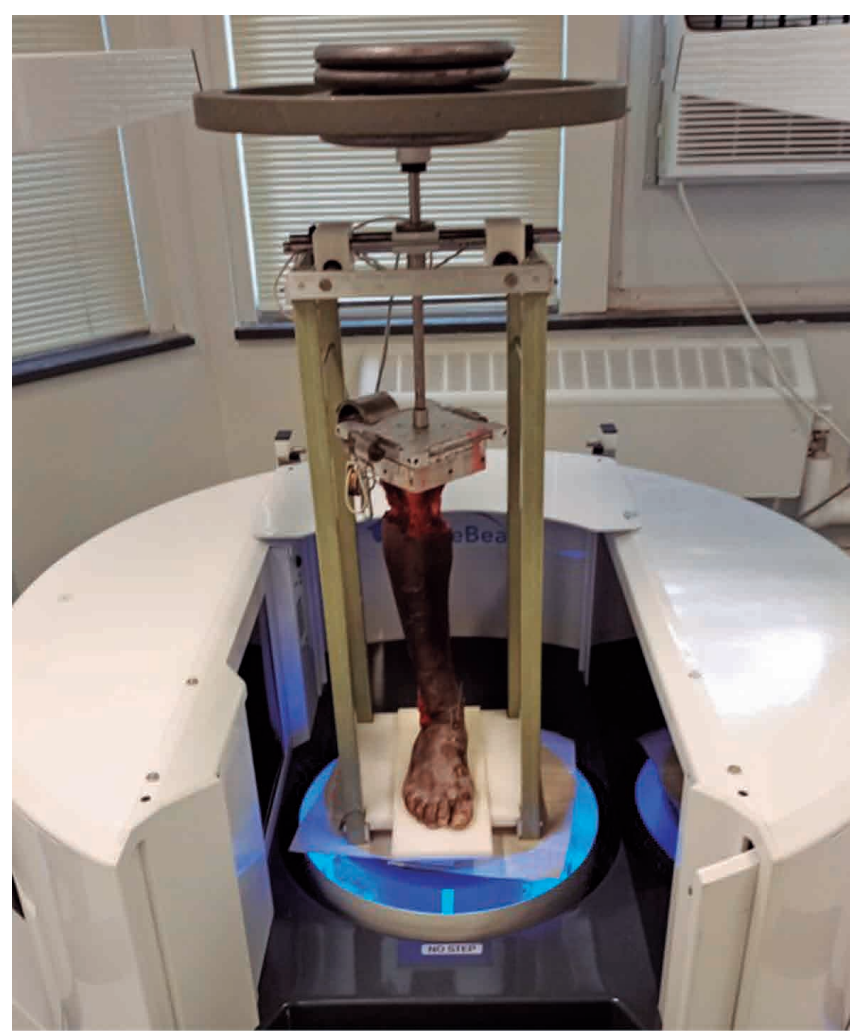

Figure 1. Overall view of the radiolucent frame, holding a leg in a simulated weight-bearing position. 


\section{First metatarsal shaft rotation}

The first metatarsal shaft coronal rotation was measured at the middle of the first metatarsal after having measured the length of the first metatarsal in the axial plane. The rotation was defined as the angle between the floor and a bisecting line of the angle formed by tangent lines to the medial and lateral surfaces of the midshaft of the first metatarsal(19) (Figure 3D).

\section{First metatarsal head coronal rotation, $\alpha$ angle}

To measure the $\alpha$ angle, a plantar line was drawn between the lateral edge of the lateral sulcus and the medial edge of the medial sulcus. A second line was drawn between the medial and lateral dorsal corners of the first metatarsal head. A third line was drawn connecting the midpoints of these 2 lines and the $\alpha$ angle was measured between this line and the vertical line ${ }^{(6)}$ (Figure 3E).
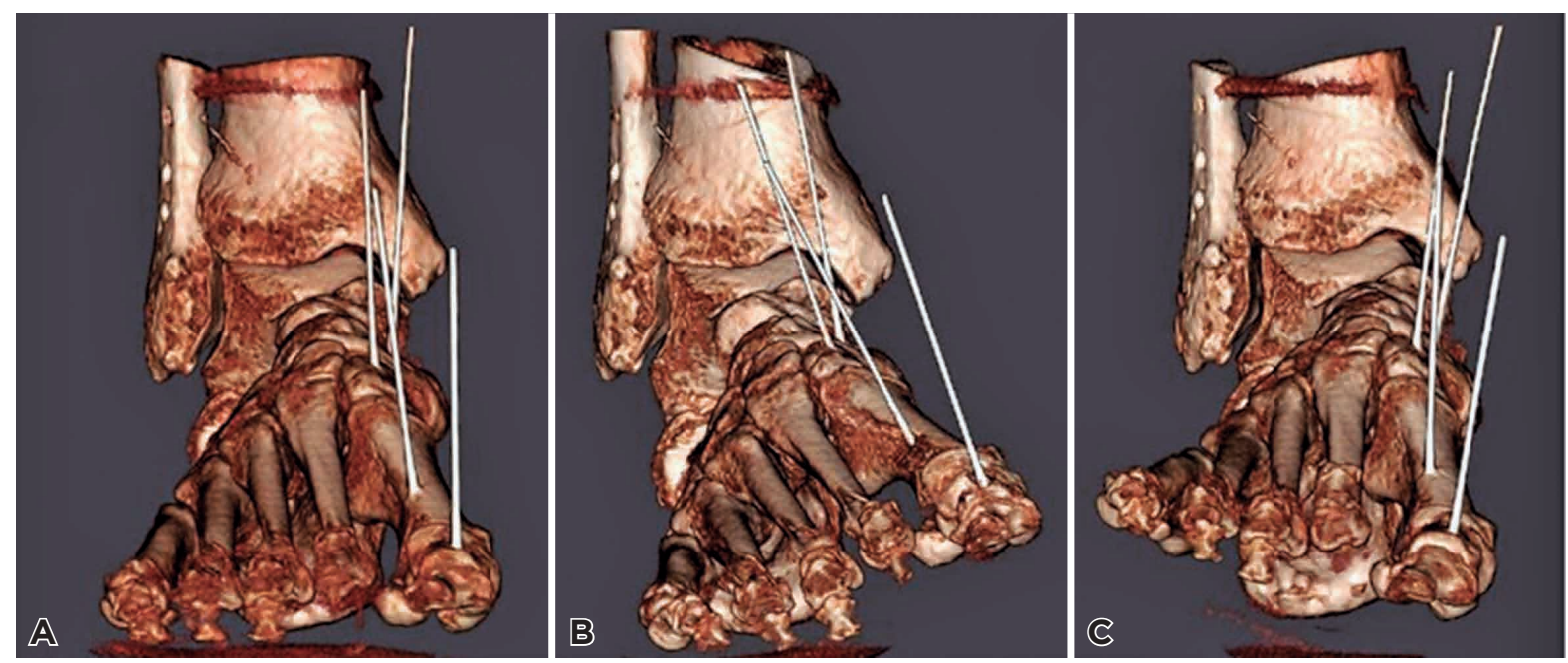

Figure 2. Three-dimensional reconstruction of weight-bearing CT acquisitions. A) Neutral condition. B) Supinated condition. C) Pronated condition.
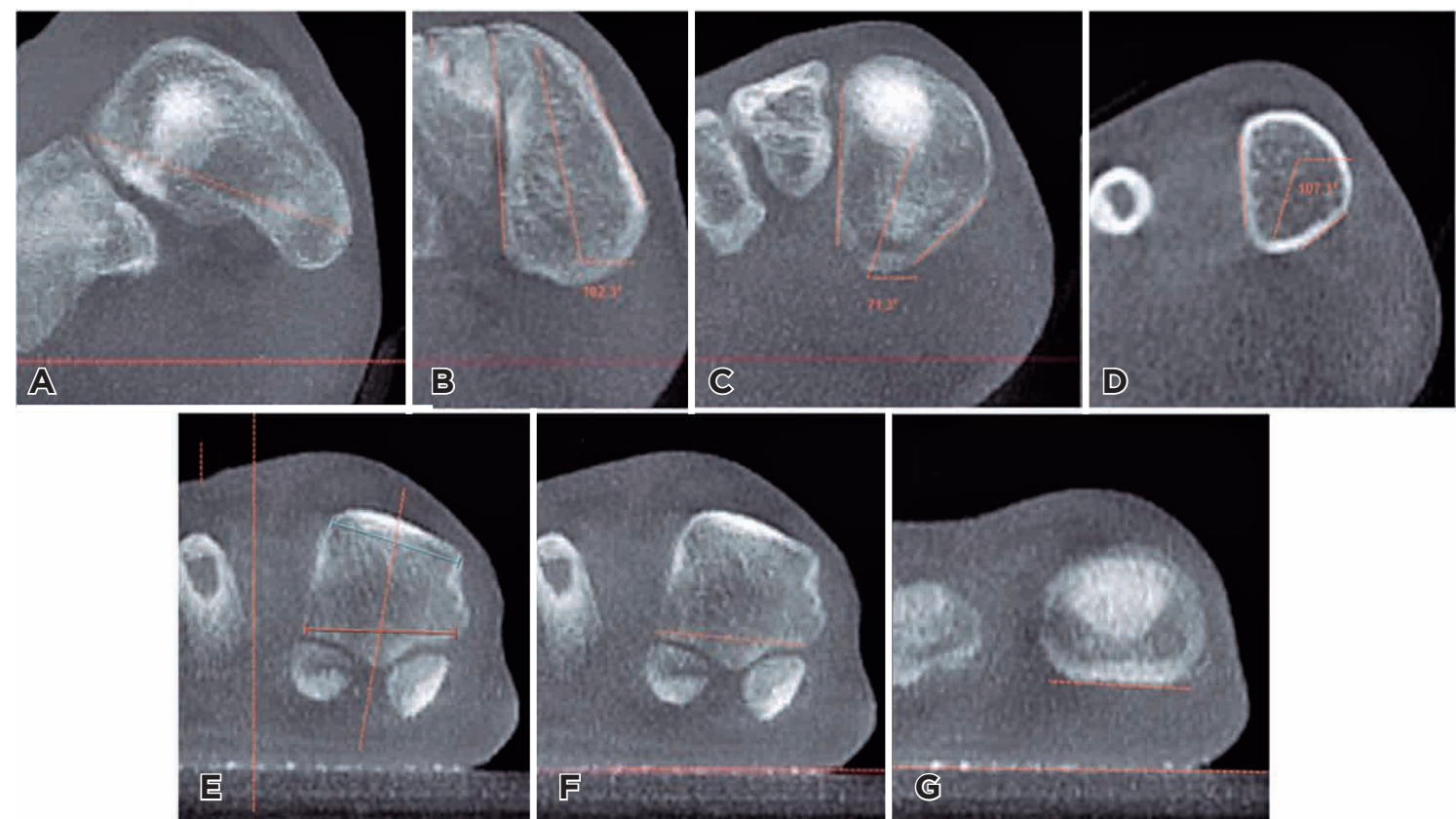

Figure 3. Illustrations of coronal plane rotation measurements of medial column bones. A) Navicular. B) Medial cuneiform. C) First metatarsal base. D) First metatarsal shaft. E) Alpha angle. F) Saltzman angle. G) Proximal phalanx of the hallux. 


\section{First metatarsal head coronal rotation, Saltzman angle}

To measure the Saltzman angle, a line was drawn touching the outer profile of the tibial and fibular sesamoid sulci at their most inferior edges. The Saltzman angle was measured between this line and the floor ${ }^{(4,18)}$ (Figure 3F).

\section{Proximal phalanx of the hallux rotation}

The proximal phalanx of the hallux rotation was measured at a level just distal to the most distal aspect of the first metatarsophalangeal joint by the angulation between the floor and a tangent line to the plantar aspect of the proximal phalanx $x^{(19)}$ (Figure 3G).

\section{Primary assessment criteria}

Two independent observers performed these 7 measurements on each specimen under each condition (neutral, supinated, and pronated), based on which we assessed interobserver reliability.

\section{Secondary assessment criteria}

To define rotational position benchmarks for each bone, the 2 observers measured the position of each pin (navicular, medial cuneiform, first metatarsal, and proximal phalanx of the hallux) relative to the vertical line in the coronal plane for each specimen under each condition (neutral, supinated, and pronated) (Figure 4A). We also assessed the interobserver reliability of these measurements. Then, the mean value of the 2 observers' measurements was taken into consideration for each pin to define the rotational position benchmarks of each bone in each condition.

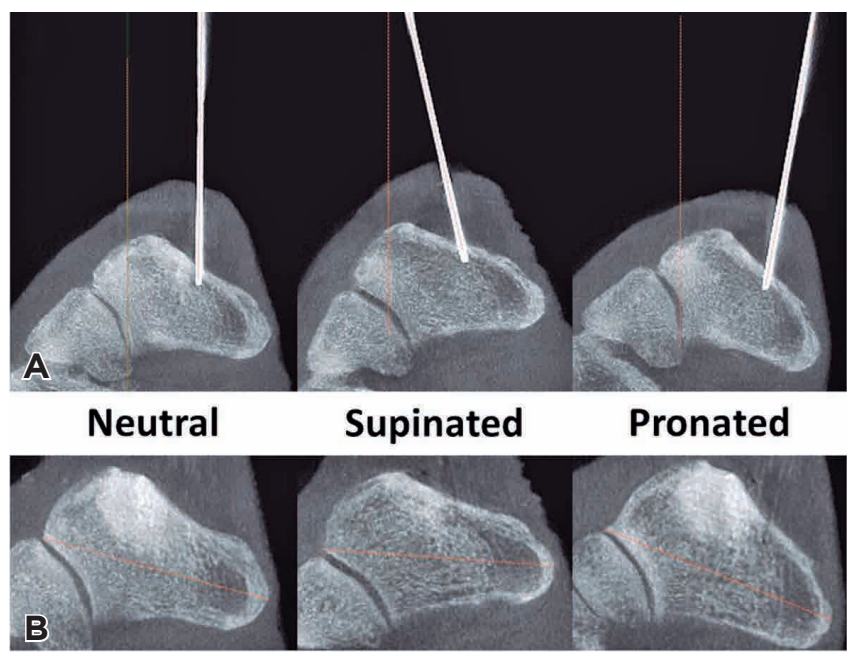

Figure 4. Assessment of the correlation between the benchmark pin and direct bone rotational measurements in the coronal plane: Example of the navicular. A) Measurements of the benchmark navicular pin rotation under neutral, supinated, and pronated conditions. B) Measurements of the navicular rotation under neutral, supinated, and pronated conditions.
We then assessed the correlation between these benchmarks and the 7 direct bone measurements performed by the 2 observers in order to assess the ability of these measurements to accurately quantify changes in bone rotation in the coronal plane (Figure 4B).

\section{Statistical analysis}

The interobserver reliability of the measurements was determined using intraclass correlation coefficients (ICCs) ${ }^{(20)}$. Pearson product-moment correlation coefficient was used to assess linear dependence between parameters. Correlation was judged to be very strong from 1 to 0.9 , strong from 0.9 to 0.7 , moderate from 0.7 to 0.5 , low from 0.5 to 0.3 , and poor from 0.3 to 0 . The $\alpha$ risk was set to 0.05 . Statistical analysis was performed with EasyMedStat (version 3.12; www.easymedstat.com).

\section{Results \\ Navicular rotation}

The interobserver reliability of the navicular rotation measurement was 0.99 (95\% confidence interval [Cl], 0.97 to 1).

The interobserver reliability of the benchmark navicular pin measurement was 1 ( $95 \% \mathrm{Cl}, 0.99$ to 1$)$. The correlations between changes in benchmark navicular pin and changes in navicular rotation direct measurement across neutral, supinated, and pronated conditions were very strong for observer $1\left(\rho=0.95 ; r^{2}=0.899 ; p=0.004\right)$ and observer $2(\rho=0.96$; $r^{2}=0.914 ; p=0.003$ ) (Figure 5A).

\section{Medial cuneiform rotation}

The interobserver reliability of the medial cuneiform rotation measurement was 0.99 (95\% $\mathrm{Cl}, 0.99$ to 1).

The interobserver reliability of the benchmark medial cuneiform pin measurement was 1 (95\% Cl, 0.99 to 1). The correlations between changes in benchmark medial cuneiform pin and changes in medial cuneiform rotation direct measurement across neutral, supinated, and pronated conditions were very strong for observer $1\left(\rho=0.98 ; r^{2}=0.97 ; p<0.001\right)$ and observer $2\left(\rho=0.98 ; r^{2}=0.96 ; p<0.001\right)$ (Figure $\left.5 B\right)$.

\section{First metatarsal base rotation}

The interobserver reliability of the first metatarsal base rotation measurement was 0.99 (95\% Cl, 0.97 to 0.99).

The interobserver reliability of the benchmark first metatarsal pin measurement was $1(95 \% \mathrm{Cl}, 0.99$ to 1$)$. This was also used as a benchmark for the first metatarsal shaft and head rotation measurements. The correlations between changes in benchmark first metatarsal pin and changes in first metatarsal base rotation direct measurement across neutral, supinated, and pronated conditions were very strong for observer 1 $\left(\rho=0.98 ; r^{2}=0.959 ; p<0.001\right)$ and observer $2\left(\rho=0.98 ; r^{2}=0.97\right.$; $\mathrm{p}<0.001$ ) (Figure $5 \mathrm{C}$ ). 


\section{First metatarsal shaft rotation}

The interobserver reliability of the first metatarsal shaft rotation measurement was 0.99 (95\% Cl, 0.99 to 1).

The correlations between changes in benchmark first metatarsal pin and changes in first metatarsal shaft rotation direct measurement across neutral, supinated, and pronated conditions were very strong for observer $1\left(\rho=0.99 ; r^{2}=0.974\right.$; $p<0.001)$ and observer $2\left(\rho=0.99 ; r^{2}=0.981 ; p<0.001\right)$ (Figure 5D).

\section{First metatarsal head coronal rotation, $\alpha$ angle}

The interobserver reliability of the $\alpha$ angle measurement was 0.99 (95\% Cl, 0.97 to 1$)$.

The correlations between changes in benchmark first metatarsal pin and changes in $\alpha$ angle across neutral, supinated, and pronated conditions were strong for observer $1(\rho=0.87$; $\left.r^{2}=0.754 ; p=0.025\right)$ and observer $2\left(\rho=0.88 ; r^{2}=0.778 ; p=0.02\right)$ (Figure 5E).

\section{First metatarsal head coronal rotation, Saltzman angle}

The interobserver reliability of the Saltzman angle measurement was 0.99 (95\% Cl, 0.98 to 1).

The correlations between changes in benchmark first metatarsal pin and changes in Saltzman angle across neutral, supinated, and pronated conditions were very strong for observer 1 $\left(\rho=0.95 ; r^{2}=0.909 ; p=0.003\right)$ and observer $2\left(\rho=0.95 ; r^{2}=0.91\right.$; $\mathrm{p}=0.003$ ) (Figure $5 \mathrm{~F}$ ).

\section{Proximal phalanx of the hallux rotation}

The interobserver reliability of the proximal phalanx of the hallux rotation measurement was 0.98 (95\% Cl, 0.92 to 0.99).

The interobserver reliability of the benchmark proximal phalanx pin measurement was $1(95 \% \mathrm{Cl}, 0.99$ to 1). The correlations between changes in benchmark proximal phalanx pin and changes in proximal phalanx of the hallux rotation direct measurement across neutral, supinated, and pronated conditions were very strong for observer $1 \quad(\rho=0.99$; $\left.r^{2}=0.992 ; \quad p<0.001\right)$ and observer $2 \quad\left(\rho=0.99 ; r^{2}=0.988\right.$; $p<0.001$ ) (Figure 5G).

\section{Discussion}

Interobserver reliability was excellent for the navicular, medial cuneiform, first metatarsal base and shaft, and proximal phalanx of the hallux rotation measurements in the coronal plane as well as for the $\alpha$ angle and the Saltzman angle. These measurements were able to accurately quantify changes in bone rotation (pronation or supination) in the coronal plane.

The reliability of the $\alpha$ angle and the Saltzman angle has already been reported as good in the literature, and our study confirmed these previous findings ${ }^{(3,4,6)}$. On the other hand, the reliability of navicular, medial cuneiform, first metatarsal base and shaft, and proximal phalanx of the hallux rotations had not been previously assessed. Najefi et al.(3) and Steadman et al. ${ }^{(4)}$ went further and defined the normative values of the $\alpha$ and Saltzman angles. Considering the excellent reliability

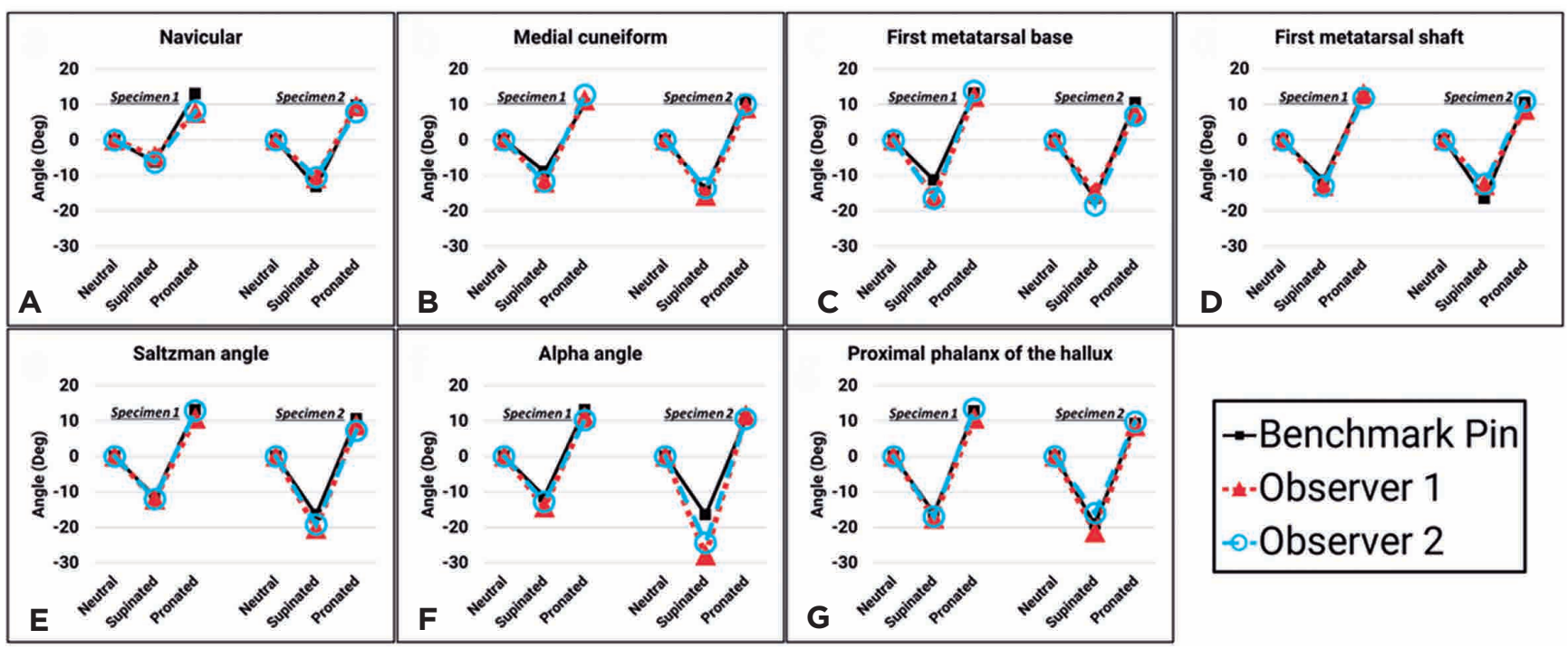

Figure 5. Charts representing the changes in rotation in the coronal plane of the benchmark pin and of the 2 observers' measurements. A) Navicular. B) Medial cuneiform. C) First metatarsal base. D) First metatarsal shaft. E) Alpha angle. F) Saltzman angle. G) Proximal phalanx of the hallux. 
of the other measurements that were performed along the medial column in our study, it would be interesting to define their normative values to serve as a reference for future studies and for the management of foot deformities. Although interobserver reliability has already been validated in the literature for the $\alpha$ and Saltzman angles, it remained uncertain whether these measurements would be able to accurately quantify changes in the rotation of the first metatarsal in the coronal plane. Saltzman et al. ${ }^{(18)}$ had previously validated the ability of the Saltzman angle to quantify rotational changes on conventional radiographs in a cadaveric study, and our study confirmed their results on WBCT acquisitions. The results of the present study showed that these measurements, together with the other measurements that were performed along the medial column, are able to accurately quantify a motion or deformity in pronation or supination in the coronal plane.

Randich et al. ${ }^{(21)}$ performed similar measurements along the medial column in the coronal plane, comparing hallux valgus and controls, but their description of some measurements lacked clarity and they did not assess the position of the navicular or perform a reliability assessment. In this context, it is challenging to evaluate the accuracy of their results. In contrast, Schmidt et al. ${ }^{(19)}$ described several measurements along the medial column in greater detail and included the navicular. Given the similarity of their intentions to ours and this contrast, we decided to perform a validation study using the descriptions provided by Schmidt et al. ${ }^{(19)}$. As described by Conti et al. ${ }^{(5)}$, the results reporting hyperpronation in hallux valgus, based on measurements performed at the level of the first metatarsal head, are an indirect reflection of an aggregate in pronation of all bones along the medial column. Therefore, using the measurements validated in the present study to compare hallux valgus and controls could reveal the exact location of this hyperpronation in the medial column in hallux valgus.

This study has several limitations. A primary limitation is that it only evaluated 2 specimens. This small sample size could not assess the impact of anatomic variability in the medial column on the measurements. Therefore, reliability values reported in this study likely represent an upper bound of the true reliability. As these specimens were selected for having no deformity, further research is needed to establish a lower bound of reliability resulting from anatomic variation. To control for these potential biases, 2 independent observers performed the measurements under 3 different conditions, and a benchmark pin was used as a control.

\section{Conclusion}

Coronal plane rotation measurements of medial column bones described in this study are reliable. These measurements can accurately quantify changes in bone rotation in the coronal plane.

Authors' contributions: Each author contributed individually and significantly to the development of this article: KD *(https://orcid.org/OOOO-OOO28061-4453) Wrote the paper, participated in the reviewing process, approved the final version; HB *(https://orcid.org/O000-0001-6917-1519) Performed acquisitions, participated in the reviewing process, approved the final version; AB *(https://orcid.org/0000-0002-4588-9291) Participated in the reviewing process, approved the final version; LMG *(https://orcid.org/0000-0001-8440-1335) Participated in the reviewing process, approved the final version; KAMC *(https://orcid.org/0000-0003-1082-6490) Participated in the reviewing process, approved the final version; NDBM *(https://orcid.org/O0000003-1067-727X) Participated in the reviewing process, approved the final version; ML *(https://orcid.org/0000-0001-5058-8867) Performed acquisitions, participated in the reviewing process, approved the final version; CCN *(https://orcid.org/0000-0001-6037-0685) Conceived and planned the activities that led to the study, approved the final version. All authors read and approved the final manuscript. *ORCID (Open Researcher and Contributor ID) (iD.

\section{References}

1. Steadman J, Barg A, Saltzman CL. First metatarsal rotation in hallux valgus deformity. Foot Ankle Int. 2021;42(4):510-22.

2. Najefi AA, Malhotra K, Patel S, Cullen N, Welck M. Assessing the Rotation of the First Metatarsal on Computed Tomography Scans: A Systematic Literature Review. Foot Ankle Int. 2021; 107110072110206

3. Najefi AA, Zaveri A, Alsafi MK, Malhotra K, Patel S, Cullen N, et al. The Assessment of First Metatarsal Rotation in the Normal Adult Population Using Weightbearing Computed Tomography. Foot Ankle Int. 2021;107110072110151.

4. Steadman J, Bakshi N, Arena C, Leake R, Barg A, Saltzman CL. Normative Distribution of First Metatarsal Axial Rotation. Foot Ankle Int. 2021;10711007211001016.

5. Conti MS, Patel TJ, Caolo KC, Amadio JM, Miller MC, Costigliola SV, et al. Correlation of Different Methods of Measuring Pronation of the First Metatarsal on Weightbearing CT Scans. Foot Ankle Int. 2021;10711007211003090.
6. Kim Y, Kim JS, Young KW, Naraghi R, Cho HK, Lee SY. A New Measure of Tibial Sesamoid Position in Hallux Valgus in Relation to the Coronal Rotation of the First Metatarsal in CT Scans. Foot Ankle Int. 2015;36(8):944-52.

7. Johnson KA, Strom DE. Tibialis posterior tendon dysfunction. Clin Orthop Relat Res. 1989;(239):196-206

8. Hardy RH, Clapham JCR. Hallux valgus; predisposing anatomical causes. Lancet. 1952;1(6720):1180-3.

9. Hardy RH, Clapham JCR. Observations on hallux valgus; based on a controlled series. J Bone Joint Surg Br. 1951;33-B(3):376-91.

10. Mansur NSB, Lalevee M, Schmidt E, Dibbern K, Wagner P, Wagner $E$, et al. Correlation between indirect radiographic parameters of first metatarsal rotation in hallux valgus and values on weightbearing computed tomography. Int Orthop. 2021;45(12):3111-8.

11. Lee HY, Lalevee M, Mansur NSB, Vandelune CA, Dibbern KN, Barg $A$, et al. Multiplanar instability of the first tarsometatarsal joint in 
hallux valgus and hallux rigidus patients: a case-control study. Int Orthop. 2021. doi: 10.1007/s00264-021-05198-9.

12. Lalevée M, Barbachan Mansur NS, Lee HY, Maly CJ, lehl CJ, Nery C, et al. Distal Metatarsal Articular Angle in Hallux Valgus Deformity. Fact or Fiction? A 3-Dimensional Weightbearing CT Assessment. Foot Ankle Int. 2021:10711007211051642.

13. Conti MS, Patel TJ, Zhu J, Elliott AJ, Conti SF, Ellis SJ. Association of First Metatarsal Pronation Correction With Patient-Reported Outcomes and Recurrence Rates in Hallux Valgus. Foot Ankle Int. 2021;107110072110469.

14. de Cesar Netto C, Godoy-Santos AL, Saito GH, Lintz F, Siegler S, O'Malley MJ, et al. Subluxation of the Middle Facet of the Subtalar Joint as a Marker of Peritalar Subluxation in Adult Acquired Flatfoot Deformity: A Case-Control Study. J Bone Joint Surg. 2019;101(20):1838-44.

15. Probasco W, Haleem AM, Yu J, Sangeorzan BJ, Deland JT, Ellis SJ. Assessment of Coronal Plane Subtalar Joint Alignment in Peritalar Subluxation via Weight-Bearing Multiplanar Imaging. Foot Ankle Int. 2015;36(3):302-9.
16. Barbachan Mansur NS, Lalevee M, Maly C, Dibbern K, Lee HY, Godoy-Santos AL, et al. Association Between Middle Facet Subluxation and Foot and Ankle Offset in Progressive Collapsing Foot Deformity. Foot Ankle Int. 2021;10711007211040820.

17. Lalevée M, Barbachan Mansur NS, Rojas EO, Lee HY, Ahrenholz SJ, Dibbern KN, et al. Prevalence and pattern of lateral impingements in the progressive collapsing foot deformity. Arch Orthop Trauma Surg. 2021. doi: 10.1007/s00402-021-04015-7.

18. Saltzman $\mathrm{CL}$, Brandser EA, Anderson CM, Berbaum KS, Brown TD. Coronal Plane Rotation of the First Metatarsal. Foot Ankle Int. 1996;17(3):157-61.

19. Schmidt E, Silva T, Baumfeld D, Dibbern K, Lee HY, Femino JE, et al. The Rotational Positioning of the Bone in the Medial Column of the Foot: A Weightbearing CT Analysis. Iowa Orthop J. 2021; 41(1):103.

20. Landis JR, Koch GG. The measurement of observer agreement for categorical data. Biometrics. 1977;33(1):159-74.

21. Randich JR, John KJ, Gomez K, Bush W. Frontal Plane Rotation of the First Ray in Hallux Valgus using Standing Computerized Tomography (CT). J Foot Ankle Surg. 2021;60(3):489-93. 